\title{
Human-Competitive Lens System Design with Evolution Strategies
}

\author{
Christian Gagné, Julie Beaulieu, Marc Parizeau* \\ Laboratoire de Vision et Systèmes Numériques (LVSN), \\ Département de Génie Électrique et de Génie Informatique, \\ Université Laval, Québec (Québec), G1K 7P4, Canada. \\ Simon Thibault \\ ImmerVision, 2020 University, Montréal (Québec), H3A 2A5, Canada.
}

\begin{abstract}
Lens system design provides ideal problems for evolutionary algorithms: a complex non-linear optimization task, often with intricate physical constraints, for which there is no analytical solutions. This paper demonstrates, through the use of two evolution strategies, namely non-isotropic SA-ES and CMA-ES, as well as multiobjective NSGA-II optimization, the human competitiveness of an approach where an evolutionary algorithm is hybridized with a local search algorithm to solve both a classic benchmark problem, and a real-world problem.
\end{abstract}

Key words: Lens Design, Global Optimization, Evolutionary Computation, Evolution Strategies, Multiobjective Optimization, Memetic Algorithm, Human Competitiveness

\section{Introduction}

Lens system design is a complex engineering task that cannot be achieved by an analytical approach. Modern optical design is conducted by experimented engineers backed by specialized CAD tools. The process starts by crafting a good initial lens system using the expert's experience and catalogs of wellknown designs. Then, refinements to the initial design are conducted using

\footnotetext{
* Corresponding author.

Email address: marc.parizeau@gel.ulaval.ca (Marc Parizeau).
} 
local search algorithms and the expert's personal experience and intuition. On the other hand, Evolutionary Algorithms (EA) consist in population-based global search methods inspired by natural evolution. They are recognized to be particularly efficient for complex non-linear optimization problems such as lens system design.

This paper is on the use of EA for automatic lens system design. Its aim is to demonstrate that the approach can lead to results comparable to those obtained by modern lens system design processes. The paper structure goes as follows. Section 2 introduces the EA with an emphasis on the algorithms used in this paper. In Section 3 some theory is given on lens system design. Section 4 is a review of different local and global computer optimization techniques for this task. Section 5 presents a lens system design benchmark problem. This problem was initially defined for a friendly competition involving human experts. Results obtained with EA for the same problem are presented in Section 6. They show that the EA used are able to find better solutions than those presented by human experts. Then, a real-world design problem for an imaging application is tackled in Section 7. Results show that EA is again able to discover lens systems that are comparable to those obtained after a reasonable effort by human experts. The same imaging problem is tackled again in Section 8, this time using a two-objective criterion to simultaneously improve image quality and reduce system cost. Finally, the paper concludes with some considerations for the automatic design of lens systems with EA.

\section{Evolutionary Computations}

Evolutionary Computations (EC) [1] encompass a family of robust search algorithms loosely inspired by natural evolution. They are a set of generic black box optimization method applicable whenever solutions can be represented by some data structure and evaluated by an objective function, the so-called "fitness" function. Populations of solutions - initially random solutions - evolve over time through a sequence of processes that include (natural) selection and different variation operations such as mutation and crossover. At the end, there is a set of good solutions to the problem and, although EC do not give any general guaranty of convergence to the global optimum, it has been shown in practice that they outperform other techniques as well as human experts for several hard problems $[2,3]$. In this project, a specific EC flavor is of interest: the evolution strategies $[1,4]$.

The evolution strategies (ES) paradigm was developed by I. Rechenberg and H.-P. Schwefel at the Berlin Technical University in the 1960s. With ES, each individual is a set of characteristics of a potential solution. This set is generally represented by a floating-point vector of fixed length. The ES is applied to 
a parent population (of size $\mu \geq 1$ ) from which individuals are randomly selected to generate a children population (of size $\lambda$ ). To engender a new population, the best $\mu$ individuals are chosen within either the $\lambda$ children (approach $(\mu, \lambda)$, where $\lambda \gg \mu$ ), or the $\mu$ parents and $\lambda$ children (approach $(\mu+\lambda)$, where $\lambda \gg 1)$. Each child is generated by a mutation of a parent, which generally consists in adding randomly generated values that follow some parameterized probability density function. In modern ES, parameters of this probability density function, often called the strategy parameters, are adapted themselves over time. Three main variants of ES with adaptive mutations are usually distinguished $[4,5]$ : $(1+1)$-ES with the $1 / 5$ rule, Self-Adaptive ES (SA-ES), and derandomized ES.

$(1+1)$-ES with the $1 / 5$ rule is the first successful ES with adaptive mutation. It consists in the evolution of a single solution with Gaussian mutation, where the mutation amplitude is adapted according to the success rate: the mutation amplitude $\sigma$ is increased if the success rate (ratio of the number of successful mutations over the number of mutations observed) is higher than $1 / 5$, the mutation amplitude is decreased if the success rate is less than $1 / 5$, and unchanged otherwise. Although the method is quite well known and has been proved to be globally convergent [6], it has the drawback of converging very slowly after an initial period of improvements [4] and to be limited by the adaptation of a global single value for all of the optimized parameter components.

In SA-ES [4], mutation parameters are associated to the individuals and are themselves changed by mutation. This coupling allow an evolution of the mutation parameters along with the optimized variables, that is an autoadaptation of the mutation strength. There are three main sub-variations of SA-ES: isotropic SA-ES, where the strategy parameter is a single value that gives the standard deviation for all components during Gaussian mutations; non-isotropic SA-ES, where the strategy parameter consists in a standard deviation value for each component during Gaussian mutations; and correlated SA-ES, where the strategy parameter of each individual is a covariance matrix parameterizing the probability density function of Gaussian mutations. In practice, non-isotropic SA-ES is generally used as it represents a good compromise between the isotropic SA-ES that restrict the feature space dimensions to a uniform scale, and the correlated SA-ES which requires very large populations to correctly estimate the covariance matrices.

Non-isotropic SA-ES are recognized as very good EA for solving real-valued parameter optimization problems. But in order to achieve good results, relatively large populations are still needed in order to have good stochastic estimates of the strategy parameters [5]. Recently, derandomized ES have been developed in order to palliate several issues of SA-ES. Similarly to the $(1+1)$ ES with the $1 / 5$ rule, they use a global parameterization of the mutation 
and a small size population. But the adaptation of this global parameterization is done by making a better use of the search path information and by smoothing adaptation over many generations. This should avoid loosing the evolution convergence, as observed with $(1+1)$-ES with the $1 / 5$ rule. Like the SA-ES, the mutation parameterization is done using several values. Currently, the Covariance Matrix Adaptation ES (CMA-ES) [7-9] is probably the most achieved derandomized ES. It consists in using a global covariance matrix to parameterize the Gaussian mutations and in adapting this covariance matrix from cumulation paths of successful mutations. CMA-ES uses a modified $(\mu, \lambda)$ strategy, that is $\left(\mu / \mu_{W}, \lambda\right)$, where $\lambda$ children are generated from a single mean parent, computed as a weighted sum of the $\mu$ parents. Just like the standard $(\mu, \lambda)$ approach, selection of individuals in the weighted $\left(\mu / \mu_{W}, \lambda\right)$ strategy is realized by keeping the $\mu$ best children, with $\lambda \gg \mu$.

Non-isotropic SA-ES and CMA-ES are used in this paper for lens system design, but many other evolutionary algorithms (e.g. differential evolution, genetic algorithms with SBX crossover, estimation of distribution algorithms) and non-evolutionary algorithms (e.g. simulated annealing) could also have been chosen for such a global optimization task. The paper should not be interpreted as a statement for the superiority of the former methods over the latter, only that the former are well adapted for the particular problem at hand. The main contribution of this paper is the methodology for automatically designing lens systems capable of human competitiveness.

\section{Theory on Lens System Design}

A lens system is an arrangement of lenses with specific refractive indices, surface curvatures, thicknesses and spacings. Figure 1 shows an example of a two-lens system. Given an object of specific size at a certain distance, its function is to produce an image of this object. Although many lens arrangements can generate images of the same size, the problem of lens system design is generally to seek the one with the least amount of aberrations. Aberrations are the difference between a real image and the corresponding approximate image computed with Gauss optics [10]. Gauss optics constitute a usable framework to characterize an optical system with various constants such as effective focal length, stop, $f$-number, and image distance and magnification. Aberrations come from the fact that Gauss optics is used during the design process; real physics of lens systems being analytically intractable.

To characterize lens systems we need to do what is called ray tracing. Starting at a given point on the object and a given initial angle, a ray trace is the computation of the trajectory of a light ray through the optical system until it reaches the image plane. The exact (real) ray trace is obtained from the first 


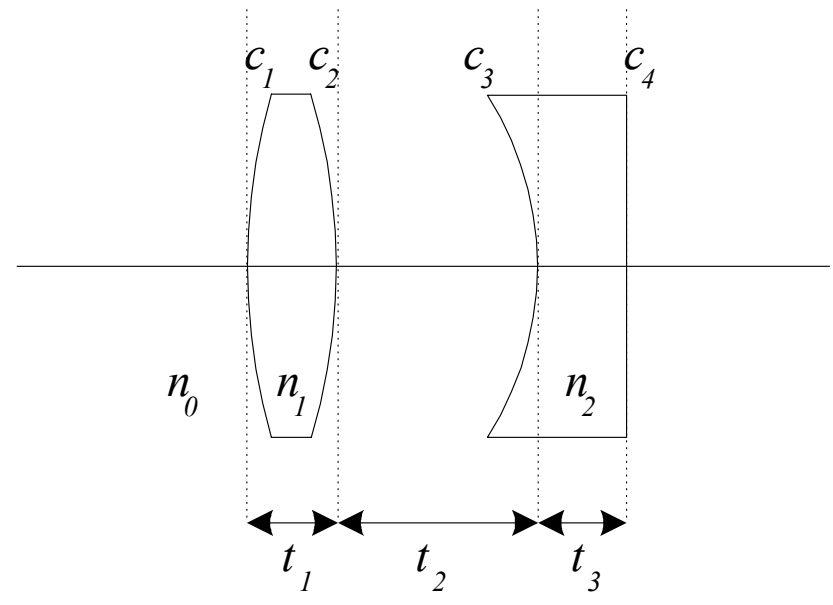

Fig. 1. Parameters of a two-lens system: $n_{j}$ represents the refractive index of a media, $c_{j}$ a lens surface curvature, $t_{1}$ and $t_{3}$ are the lens thicknesses, and $t_{2}$ is the lens spacing.

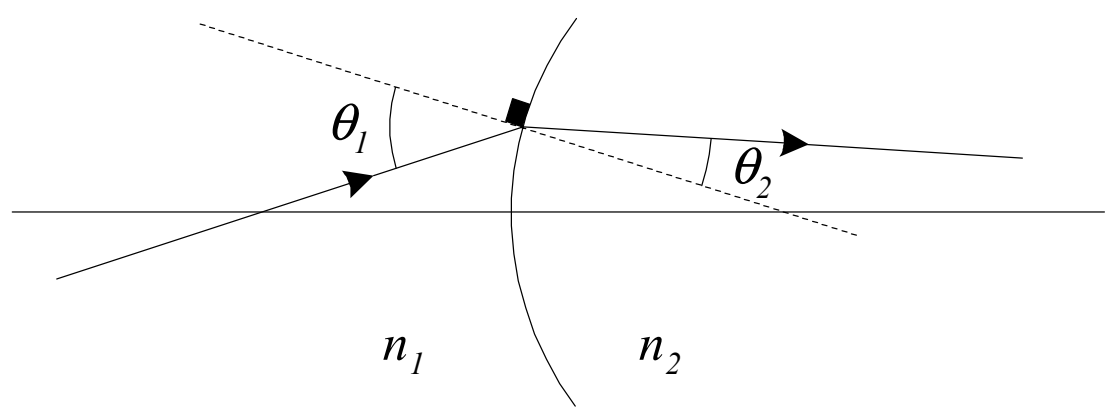

Fig. 2. Illustration of Snell-Descartes first law of refraction.

law of refraction (Snell-Descartes) that governs the behavior of light passing through the interface between two media having different refractive indices. The path of a ray passing from medium 1 to medium 2 obeys the following equation:

$$
n_{1} \sin \theta_{1}=n_{2} \sin \theta_{2}
$$

where $n_{1}$ and $n_{2}$ are refractive indices of media 1 and 2, and $\theta_{1}$ and $\theta_{2}$ are incident and refracted angles relative to the normal of the interface between the two media. Figure 2 illustrates this first law of refraction. On the other hand, the paraxial approximation consists in assuming that all rays lie close to the optical axis. Using the sine expansion:

$$
\sin \phi=\phi-\frac{\phi^{3}}{3 !}+\frac{\phi^{5}}{5 !}-\cdots
$$

and let $\phi \approx 0 \Longrightarrow \sin \phi \approx \phi$. Equation 1 becomes:

$$
n_{1} \theta_{1} \approx n_{2} \theta_{2}
$$




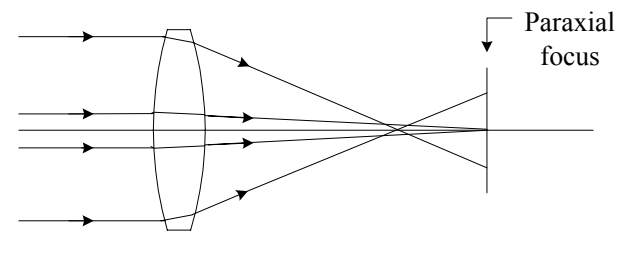

a)

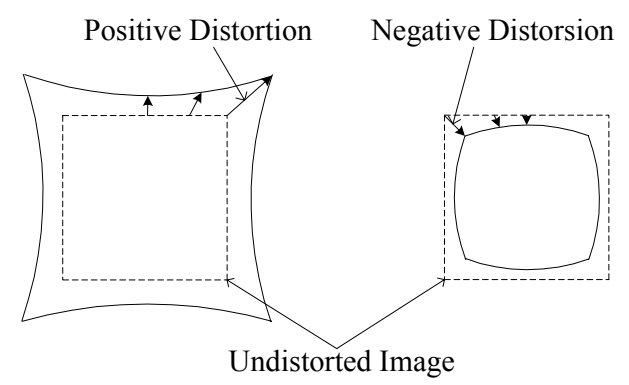

b)

Fig. 3. Two of the Seidel aberrations: a) spherical aberration, and b) distortion.

This approximation is the basis of Gauss optics or first order optics.

The aberrations of an optical system are measured by computing the difference between the real image (i.e. the one that stems from Equation 1), and the image that results from the paraxial approximation. In other words, two ray traces emerging from the same point on the object with the same (non-zero) angle, one exact and one approximated ${ }^{1}$, will strike the image plane at different positions. These differences, averaged over a whole set of distinct rays provide a convenient basis for building a quality measure.

It is interesting to note that if we also consider the second term of the sine expansion in Equation 2, we obtain what is called third order optics. The difference between first and third order optics represents the five Seidel aberrations: spherical aberration, coma, astigmatism, field curvature, and distortion [10]. Figure 3 illustrates two of these. The spherical aberration (Figure 3a) is caused by the fact that, for spherical lenses, rays coming from infinity and parallel to the optical axis do not converge to the same focus point, depending on the ray distance from the optical axis. The result of this type of aberration is a blurred image. Another type of aberration is distortion, that causes pincushion (positive distortion) or barrel (negative distortion) shaped images, as shown in Figure 3b.

Finally, it should be noted that the refractive index of a given glass is not constant but varies as a function of the light wavelength. The refractive index value found in the literature is usually the refractive index value of the material at the Helium $d$ wavelength $(\lambda=587.6 \mathrm{~nm})$. Also, the refractive index rate of change with the wavelength is different from one glass to another. It is standard to characterize the dispersion property of a given glass using a measure called the $v$-number (or Abbe number). This measure is simply a relative rate of change of the refractive index, calculated using the refractive index of the material at three arbitrary wavelengths. The $v$-number of glasses is a factor that should be taken into account when designing polychromatic lens systems.

$\overline{1}$ The approximated ray trace is virtual and computed with Gauss optics. 


\section{Lens System Optimization}

Modern design of lens systems is generally conducted using specialized CAD software that help designers to visualize the lens system, to evaluate its quality based on precise criteria, and to locally optimize the system variables. This optimization is often achieved by local search algorithms such the Damped Least Squares (DLS) method. But the typical search space of optical system design is a complicated multidimensional space comprising several peaks, non-linearities and strong correlation between parameters [11]. Hence, a local search explores only the immediate neighborhood of the initial solution, making the result very dependent on the starting point solution. But since the end of the 1980's, several applications of global search methods have been made in optical design. A few researchers have successfully used simulated annealing $[12,13]$. Others have modified local optimization algorithms, like the DLS algorithm, to allow exploration beyond local optima [14]. These two approaches have been recently integrated in some optical CAD tools.

The idea of using EA as a global optimization method for lens system design has been investigated independently by several researchers. The pioneering work of Walk and Niklaus [15] consists in the application of a basic ES to lens system design. Betensky [16] presents an original method incorporating a set of zero power operators that do not change significantly the first order properties of lens systems. Bit string Genetic Algorithms (GA) $[17,18]$ were used, with each position in the string representing the application (or not) of the associated zero power operator. Each design modified by these operators is re-optimized with traditional local search algorithms. Since 1996, several papers have been published on the use of bit string GAs [19,20], real-valued GA $[21-23]$ or ES $[22,24]$ for the optimization of a fixed number of real-valued parameters. Results presented in [21] are apparently very good, with the successful automatic design of large-scale lens systems composed of more than ten parts with real-valued GA, and experiments on the use of Pareto optimal selection strategy for multiobjective optimization [25]. Others [26,27] have used a two step approach, starting with a bit string GA global search to find a good starting point solution, then using DLS optimization and the end of the evolution to further refine the best GA-found solution. Unfortunately, there is no baseline results generated by human experts or other automatic approaches presented in these papers, for which comparative results would be good indicators on the real capability of EC for fully automated lens system design.

In [28], we presented results on the automatic lens system design with EA for a benchmark problem introduced in [29]. Since the publication of that paper, Nagata [30] gave some results for the same benchmark problem and other problems taken from [21] using the CMA-ES algorithm. In his paper, Nagata 
used its own quality measure, with no clear statement on the superiority of its results compared to those of [29] and [28].

Recently, Koza, Al-Sakran and Jones [31,32] presented a developmental genetic programming approach [33] for optical system design with a variable number of lenses. They tested their approach on six patented lens systems, with lens systems automatically generated by GP using the prescription given in the patent statement as framework for the fitness function. Their evolutions were conducted on very big population (respectively 346000 and 75000 individuals) and the lens systems obtained are apparently of performance level comparable to those patented. No local optimization were applied to finely tune the numeric parameters of the lens systems.

The present paper is an extension of our previous paper [28], where different evolutionary optimization techniques were tested. The main contribution of the present paper is to detail an approach based on state-of-the-art EA for real-valued optimization, namely SA-ES and CMA-ES, which are hybridized with local search algorithm specific to lens system design. Results obtained for the benchmark problem and a new real world problem are comparable and sometimes even better than those produced by optical experts, hence showing the human-competitiveness of the approach. These results are of great importance, as we clearly demonstrates the practical viability of EA to further automate lens system design.

\section{Monochromatic Quartet}

For the first experiments on evaluating the capability of EA for automatic lens system design, we choose a problem defined for the 1990 International Lens Design Conference (ILDC 1990). This conference, held every four years, includes a friendly lens design competition for its participants. The 1990 problem [29] became a benchmark to evaluate the performance of optimization algorithms for lens system design because the 11 best solutions proposed by human experts form only two different classes of similar solutions, and the organizers concluded that these solutions were probably global optimums of the solution space.

This benchmark problem is named the monochromatic quartet. Essentially, it consists in finding an optical system made of four spherical lenses. Here is the formal statement of the problem [29]:

Design a 4-element, f/3, $100 \mathrm{~mm}$ effective focal length lens of BK'7 glass, illuminated by helium $d$ wavelength (i.e., $n=1.51680$ ). The object is at infinity, the object field covers $30^{\circ}$ full field $\left(15^{\circ}\right.$ semi-field angle) and the 
image field is flat.

Constraints on the construction includes: only spherical surfaces, no aspherics, GRIN elements, Fresnel lenses, binary elements, holographic optical elements, etc. The minimum glass thickness is $2 \mathrm{~mm}$, but there is no upper limit on the size of the lens. The distortion must be less than $1 \%$ and there should be no vignetting. The last is intended to assure that vignetting could not be used to improve the edge performance on the lens. No requirement is put on the location of the stop of the system.

The merit function consists of the average of the RMS blur spot for three fields : on-axis, $10.5^{\circ}$, and $15^{\circ}$, weighted equally.

The $f$-number (also written $f / \#$ ) is a measure of the size and light-collecting ability of the lens system. The effective focal length for a lens system is similar to the focal length of an equivalent single lens, which is the distance from the center of the lens to the convergence point of rays that are initially parallel to the optical axis. The BK7 glass is just an ordinary type of glass frequently used for lens fabrication. The helium $d$ wavelength constraint specifies that the problem is monochromatic, that is the considered wavelength is fixed and thus the refractive index is also fixed (otherwise we would have to consider different refractive indices for different wavelengths). The system must not have vignetting, i.e. the image must not be truncated. It is also possible to include a stop, that is an aperture in the optical system which limits the amount of light in the system, allowing to reduce aberrations. Its diameter directly influences the effective focal length and the $f$-number.

The problem is formulated in such a way that the error measure of distortion is separated from the other aberrations. The problem statement specifies that distortion must not exceed $1 \%$ and thus implies that below this level, one should only concentrate on minimizing other aberrations. Using exact computations (Equation 1), the RMS blur spot method consists in tracing several parallel rays at a given entrance angle. These angles must be set successively at $0^{\circ}, 10.5^{\circ}$, and $15^{\circ}$ as specified in the problem statement. Using paraxial approximation, all the rays with the same entrance angle converge at a single point. But with exact ray traces, they will strike the image plane at different points, generally in the neighborhood of the approximated point, and form a so-called blur spot, as illustrated in Figure 4. The RMS blur spot size is computed from the variance of the position at the image plane of different exact rays with the same entrance angle. A reference ray traced with the paraxial approximation is used to evaluate the distortion, measuring its distance from the centroid of the exact rays at the image plane.

Figure 5 presents two of the best lens designs presented at ILDC 1990, one of each class of similar solutions. The first is solution \#14, the best overall reported solution, and the second is solution \#7, the best for the second class of designs (4th overall). RMS blur spot sizes computed with the commercial 


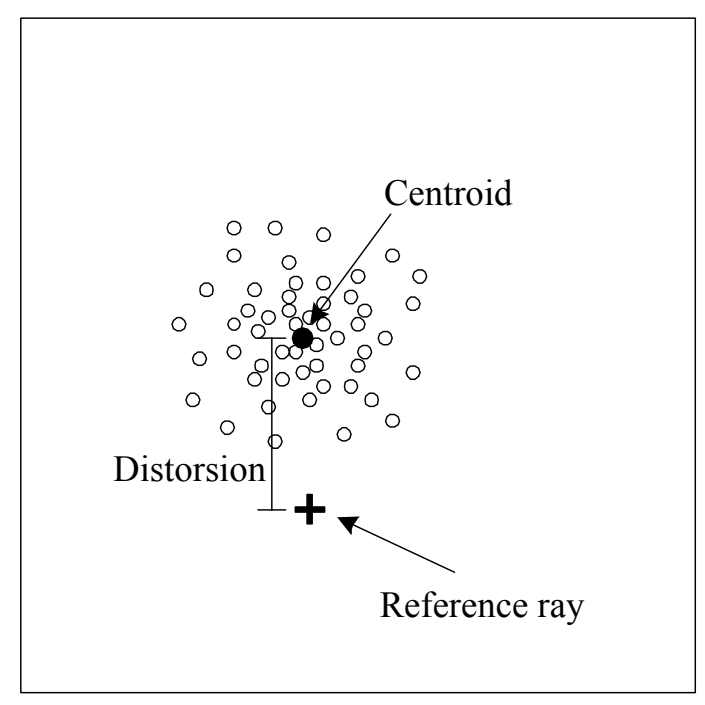

Fig. 4. Illustration of the distortion measure with the blur spot centroid.

CAD tool CODE V [34] are respectively $0.0022 \mathrm{~mm}$ and $0.0025 \mathrm{~mm}$. These are slightly different from those reported in [29] (0.0021 $\mathrm{mm}$ and $0.0024 \mathrm{~mm})$, which were computed using a different software.

\section{Evolutionary Monochromatic Quartet}

In optimization problems, one needs to consider the exploration vs exploitation dilemma. The configuration of the search algorithm should make a good tradeoff between exploring unvisited regions of the search space, in order to discover better solutions, and exploiting known regions that are rich in good solutions. For the specific problem of lens systems, local search algorithms such as DLS are very good to discover local optima. EA should thus be used mostly for the exploration part of the optimization algorithm, letting exploitation to well established numerical methods. Much like [16], but contrary to $[26,27]$ where local optimization is conducted at the end of evolutions, we apply local optimization to each individual of each generation, provided that it satisfies the problem constraints. This is an hybrid approach, sometimes called memetic algorithms [35,36], that has the advantage of reducing the exploitation pressure on the EA, favoring more exploratory searches.

For the monochromatic quartet, individuals can be represented as a vector of 15 real-valued parameters. During initialization, each parameter is uniformly generated in the $[-1,1]$ interval. Each parameter value is then scaled at the fitness evaluation, based on its respective search bounds. Table 1 summarizes the parameters with their respective value transformations. The last surface curvature, the distance between the last surface and the image plane, and the stop aperture are dependent variables, computed for each system in order 


\begin{tabular}{cccc}
\hline $\begin{array}{c}\text { Radius } \\
(\mathrm{mm})\end{array}$ & $\begin{array}{c}\text { Thickness } \\
(\mathrm{mm})\end{array}$ & $\begin{array}{c}\text { Semi- } \\
\text { Aperture }\end{array}$ & Glass \\
\hline 0.0 & $\infty$ & - & AIR \\
145.6875 & 2.0 & 64.9691 & BK7 \\
94.7116 & 10.8039 & 61.6892 & AIR \\
162.7274 & 231.4971 & 61.6778 & BK7 \\
-143.6539 & 0.0 & 15.3583 & AIR \\
$\mathbf{0 . 0}$ & $\mathbf{0 . 1}$ & $\mathbf{1 3 . 5 6 7 4}$ & AIR \\
68.4004 & 103.2294 & 14.3693 & BK7 \\
-1480.2792 & 6.7215 & 23.2095 & AIR \\
-43.0337 & 2.0 & 23.2221 & BK7 \\
858.3420 & 2.0046 & 25.8911 & AIR \\
0.0 & - & 26.7948 & - \\
\hline \multicolumn{4}{c}{ Bold surface is the aperture stop. $)$}
\end{tabular}

a)

\begin{tabular}{cccc}
\hline $\begin{array}{c}\text { Radius } \\
(\mathrm{mm})\end{array}$ & $\begin{array}{c}\text { Thickness } \\
(\mathrm{mm})\end{array}$ & $\begin{array}{c}\text { Semi- } \\
\text { Aperture }\end{array}$ & Glass \\
\hline 0.0 & $\infty$ & - & AIR \\
116.753325 & 8.0 & 41.3577 & BK7 \\
-896.302517 & 4.6 & 41.5202 & AIR \\
-148.252719 & 86.666308 & 41.7531 & BK7 \\
-170.784756 & 0.0 & 23.9590 & AIR \\
61.852545 & 22.856198 & 21.7151 & BK7 \\
$\mathbf{0 . 0}$ & $\mathbf{6 6 . 1 8 7 9 6 1}$ & $\mathbf{1 1 . 5 9 1 0}$ & BK7 \\
-406.404293 & 5.8 & 19.5193 & AIR \\
-36.156468 & 3.965152 & 19.5915 & BK7 \\
-1470.14649 & 0.0 & 22.4919 & AIR \\
0.0 & - & 26.7948 & - \\
\hline \multicolumn{4}{c}{ Bold surface is the aperture stop. $)$}
\end{tabular}

b)

Fig. 5. Best lens systems of two classes of designs presented by human experts at ILDC 1990: a) solution 14 (blur spot of $0.00218 \mathrm{~mm}$ ) and b) solution 7 (blur spot of $0.00250 \mathrm{~mm}$ ).

to get the paraxial image in-focus on the image plane, and to satisfy the specified $f$-number (3.0) and effective focal length (100.0). The stop location is the relative position of the stop between the first and the last surface of the system. Lens curvature is simply the inverse of lens radius $\left(c_{j}=1 / r_{j}\right)$.

The fitness measure used for the experiments is composed of a scalar fitness value and a Boolean stating whether the solution is feasible or not. If the original system is not feasible, the fitness value is computed from the sum of Seidel aberrations plus some constraint penalties: 
Table 1

Number of parameters and value transformations for the monochromatic quartet. Tilde variables (i.e. $\tilde{x}$ ) represent untransformed vector components.

\begin{tabular}{ccc}
\hline Type & \# of Param. & Value Transformation \\
\hline Curvature & 7 & $c_{j}=0.025 \tilde{c}_{j} \mathrm{~mm}^{-1}$ \\
Thickness & 4 & $t_{j}=\left(\left|10 \tilde{t}_{j}\right|+2\right) \mathrm{mm}$ \\
Distance & 3 & $t_{j}=\left|10 \tilde{t}_{j}\right| \mathrm{mm}$ \\
Stop location & 1 & $s=\left\{\begin{array}{cc}|\tilde{s}| \tilde{s} \in[-1,1] \\
1 & \text { otherwise }\end{array}\right.$ \\
\hline
\end{tabular}

Table 2

Physical constraints for the monochromatic quartet; the different penalties are null when the constraint is satisfied. \% dist is the percentage of distortion measured on the image plane, and $l_{\text {vign }}$ is the number of surfaces affected by vignetting.

$$
\begin{array}{ccc}
\hline \text { Type } & \text { Constraint } & \text { Penalty } \\
\hline \text { Distortion } & \left|\%_{\text {dist }}\right| \leq 1 & P_{\text {dist }}=\left|\%_{\text {dist }}\right| \\
\text { Image distance } & t_{\text {img }} \geq 0 & P_{t_{\text {img }}}=-t_{\text {img }} \\
\text { Vignetting } & l_{\text {vign }}=0 & P_{\text {vign }}=1000 l_{\text {vign }} \\
\text { RMS blur spot } & \text { at } 0^{\circ}, 10.5^{\circ}, \text { and } 15^{\circ} & P_{\mathrm{RMS}}=1000 \\
\hline F_{1}=1000+B+F+C+P I^{2}+E & \\
+P_{\text {dist }}+P_{t_{\text {img }}}+P_{\text {vign }}+P_{\mathrm{RMS}} &
\end{array}
$$

where $B, F, C, P I^{2}$, and $E$ are the five Seidel aberrations, respectively spherical aberration, coma, astigmatism, field curvature, and distortion, and the $P_{x}$ are different penalties computed from a set of physical constraints, as presented in Table 2. The arbitrary penalty value of 1000 in Equation 4 is used to make sure that the fitness values of unfeasible designs are always worse than those of feasible ones.

If the original system is feasible, then the fitness value depends only on the average RMS blur spot size which is computed for the three field angles:

$$
F_{2}=\frac{1}{3} \sum_{\theta=\{0,10.5,15\}} \operatorname{RMS}_{\theta}^{\text {orig }}
$$

Local optimization is next applied to the system for a period of up to six seconds. Preliminary experiments have shown that this delay is more than enough to reach a local optimum, in most circumstances. If this local optimum is also feasible, a new average RMS blur spot size is computed from the 
optimized solution.

$$
F_{3}=\frac{1}{3} \sum_{\theta=\{0,10.5,15\}} \operatorname{RMS}_{\theta}^{\mathrm{opt}}
$$

The final fitness value is thus $F_{1}, F_{2}$, or $F_{3}$ depending on the context.

The use of distinct fitness measures $F_{1}$ and $F_{2}$ for unfeasible and feasible lens system evaluation is motivated by the brittleness of the RMS blur spot size quality criterion which cannot always be computed by the CAD tool for the three specified field angles. In those cases, an alternate criterion based on the sum of Seidel aberrations is used instead.

For all experiments presented in this paper, the Open BEAGLE [37] C++ framework $^{2}$ was used to implement the corresponding EA. The lens system characteristics (Seidel aberrations, RMS blur spot size, etc.) were computed using the commercial CAD tool CODE V [34]. This tool is also used to apply the six seconds of local optimization using the DLS algorithm ${ }^{3}$.

As a first experiment, a $(\mu+\lambda)$ non-isotropic SA-ES was applied to the monochromatic quartet problem. Five populations of $\mu=50$ individuals each were used, with $\lambda=350$ children generated at each generation for each population using the usual SA-ES mutation. One-way random ring migration was also applied at each generation using two individuals chosen from each population. Runs were conducted over 250 generations, with initial strategy parameters of $\sigma_{j}=2.0$, and a lower bound value of 0.05 . This experiment was repeated five times, and the best result is presented in Figure 6. Its RMS blur spot size is $0.00167 \mathrm{~mm}$, that is $23 \%$ smaller than the best human-engineered design presented at ILDC 1990. But the design is also very peculiar. Indeed, its total track (length of assembly) is more than 23 meters long and the first lens is disproportionate compared with the others. Nevertheless, it satisfies all of the problem constraints. To our knowledge, it is the best design ever for this problem, and contradicts the belief that the two design classes presented at ILDC 1990 are global optima. Another interesting point is that the five independent runs of $(\mu+\lambda)$ non-isotropic SA-ES converged toward the same design class.

As a second experiment, CMA-ES was also applied to the monochromatic quartet problem, but using a single population of $\mu=10$ individuals, $\lambda=50$ children, and 500 generations. Figure 7 presents the best lens design obtained over the five runs. This result obtained with CMA-ES belongs to the second design class of solutions presented at ILDC 1990 (Figure 5b). At $0.00393 \mathrm{~mm}$,

$\overline{2}$ Freely available at http://beagle.gel.ulaval.ca.

3 Using the command AUT with global optimization deactivated (GS NO). 


\begin{tabular}{cccc}
\hline $\begin{array}{c}\text { Radius } \\
(\mathrm{mm})\end{array}$ & $\begin{array}{c}\text { Thickness } \\
(\mathrm{mm})\end{array}$ & $\begin{array}{c}\text { Semi- } \\
\text { Aperture }\end{array}$ & Glass \\
\hline 0.0 & $\infty$ & - & AIR \\
11141.72266 & 20296.860683 & 9185.5313 & BK7 \\
184.34870 & 110.757080 & 153.1824 & AIR \\
-487.56700 & 2438.208956 & 151.9749 & BK7 \\
$\mathbf{0 . 0}$ & $\mathbf{5 . 9 9 6 6 2 7}$ & $\mathbf{4 9 . 4 4 4 1}$ & BK7 \\
-206.83954 & 8.574215 & 49.4441 & AIR \\
224.63126 & 4.437700 & 47.4147 & BK7 \\
1803.40918 & 5.638265 & 47.4147 & AIR \\
-226.65711 & 200.040559 & 47.4147 & BK7 \\
-234.83769 & 211.117756 & 43.9963 & AIR \\
0.0 & - & 26.7949 & - \\
\hline \multicolumn{4}{c}{ (Bold surface is the aperture stop. $)$} \\
\hline
\end{tabular}

Fig. 6. Best lens system found with $(\mu+\lambda)$ non-isotropic SA-ES for the monochromatic quartet problem (RMS blur spot size of $0.00167 \mathrm{~mm}$ ).

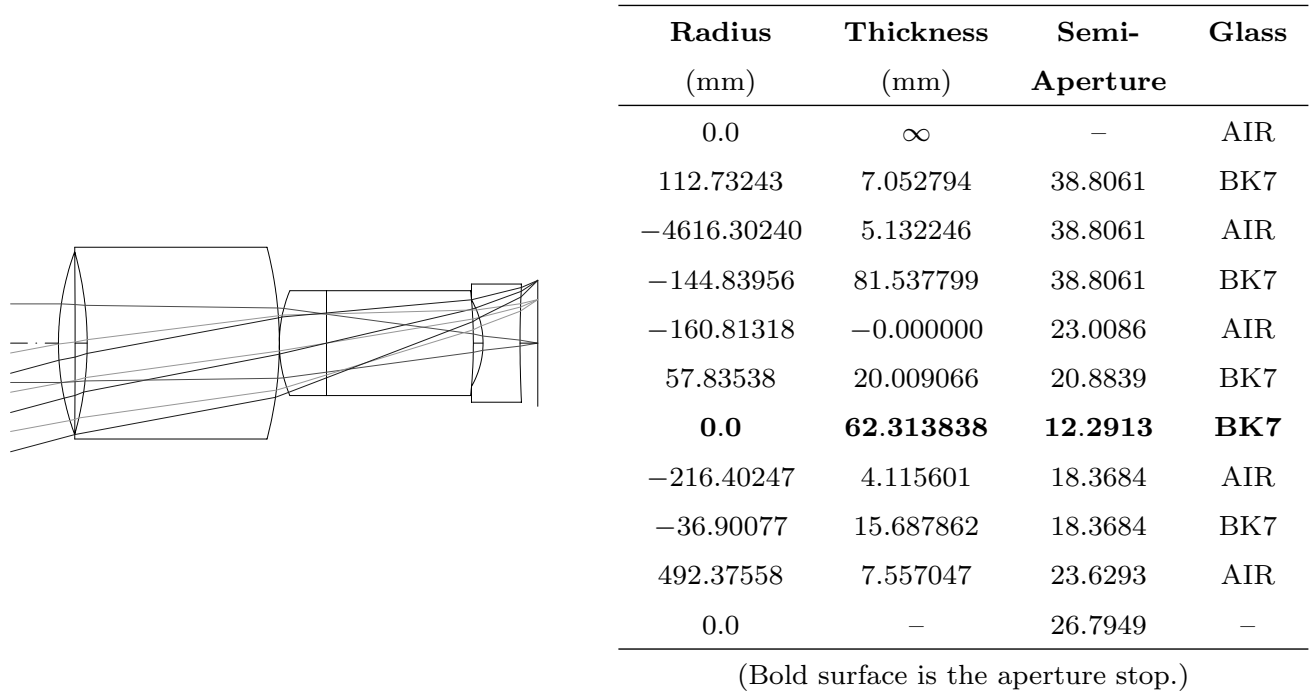

Fig. 7. Best lens system found with CMA-ES for the monochromatic quartet problem (RMS blur spot size of $0.00393 \mathrm{~mm}$ ).

its blur spot size is $57 \%$ worse than the corresponding best human-engineered design of the same class, but still a lot better than the worse qualified human design presented at ILDC 1990 (solution 4 with a blur spot size of $0.00605 \mathrm{~mm}$ according to our measures). It should be noted that computational resources deployed for this solution were more than 10 times lower than with SA-ES (about 15 hours vs 167 hours using a $3 \mathrm{GHz}$ Pentium 4 PC). The difference between the two algorithms stems from the large population size required by $(\mu+\lambda)$ non-isotropic SA-ES, which increases the computational requirements, but also allows a finer solution space exploration. On the other hand, CMA-ES is more computationally efficient, at the cost of a less systematic exploration of the search space. 
Table 3

Specifications of the ILS problem.

\begin{tabular}{cc}
\hline Specification & Requirement \\
\hline Number of lenses & $\leq 5$ \\
Total track length & $\sum t_{j} \leq 120 \mathrm{~mm}$ (object up to image) \\
Object distance & $t_{\mathrm{obj}}=75 \mathrm{~mm}$ \\
Full field of view & $80 \mathrm{~mm}\left(y_{\mathrm{obj}} \in[-40,40]\right)$ \\
Magnification & $m=-0.36$ \\
$f$-number & $f / 4($ to be determined) \\
Vignetting & No vignetting tolerated. \\
Lens semi-aperture & $a_{j} \leq 15 \mathrm{~mm} \quad \forall j$ \\
Wavelengths and weights & $865 \mathrm{~nm} \times 0.5,890 \mathrm{~nm} \times 1.0,915 \mathrm{~nm} \times 0.5$ \\
Image format & $\mathrm{CCD}$ detectors of $15 \times 15 \mu \mathrm{m}$ \\
Image quality & $75 \%$ encircled energy diameter less than $15 \mu \mathrm{m}$ \\
\hline
\end{tabular}

\section{Imaging Lens System}

In this section, a real-world application, the imaging lens system (ILS) problem is tackled. This problem was presented to the optical design team of the National Optics Institute (INO), in Quebec City. The INO experts found a solution that satisfied the specification constraints after conducting a best effort search within the budget limits defined by the client: about 5 man-days. No doubt that with more resources, these experts could have produced a better system.

The problem is to design an imaging system of limited length. The detailed specifications are given in Table 3. This problem has strong physical constraints that are difficult to satisfy. The target imaging quality criterion is the $75 \%$ encircled energy diameter, with a primary target of making this circle fit into a $15 \times 15 \mu \mathrm{m}$ CCD sensor. Smaller encircled energy diameters means better image quality. The $75 \%$ encircled energy diameter is evaluated using three wavelengths of different weights as specified in Table 3. The requirement on the $f$-number is an indication rather than a strict specification: the working $f$-number of the resulting system may be slightly modified if necessary.

Figure 8 describes the design that was developed by INO experts. It satisfies all of the constraints of the problem. The $75 \%$ encircled energy diameter is 13.3 $\mu \mathrm{m}$ for points at $0 \mathrm{~mm}$ on the object plane, $33.0 \mu \mathrm{m}$ for points at $28 \mathrm{~mm}$, and $21.8 \mu \mathrm{m}$ for points at $40 \mathrm{~mm}$ on the object plane. Since the specification for the image quality is somewhat fuzzy and the $75 \%$ encircled energy diameter is 


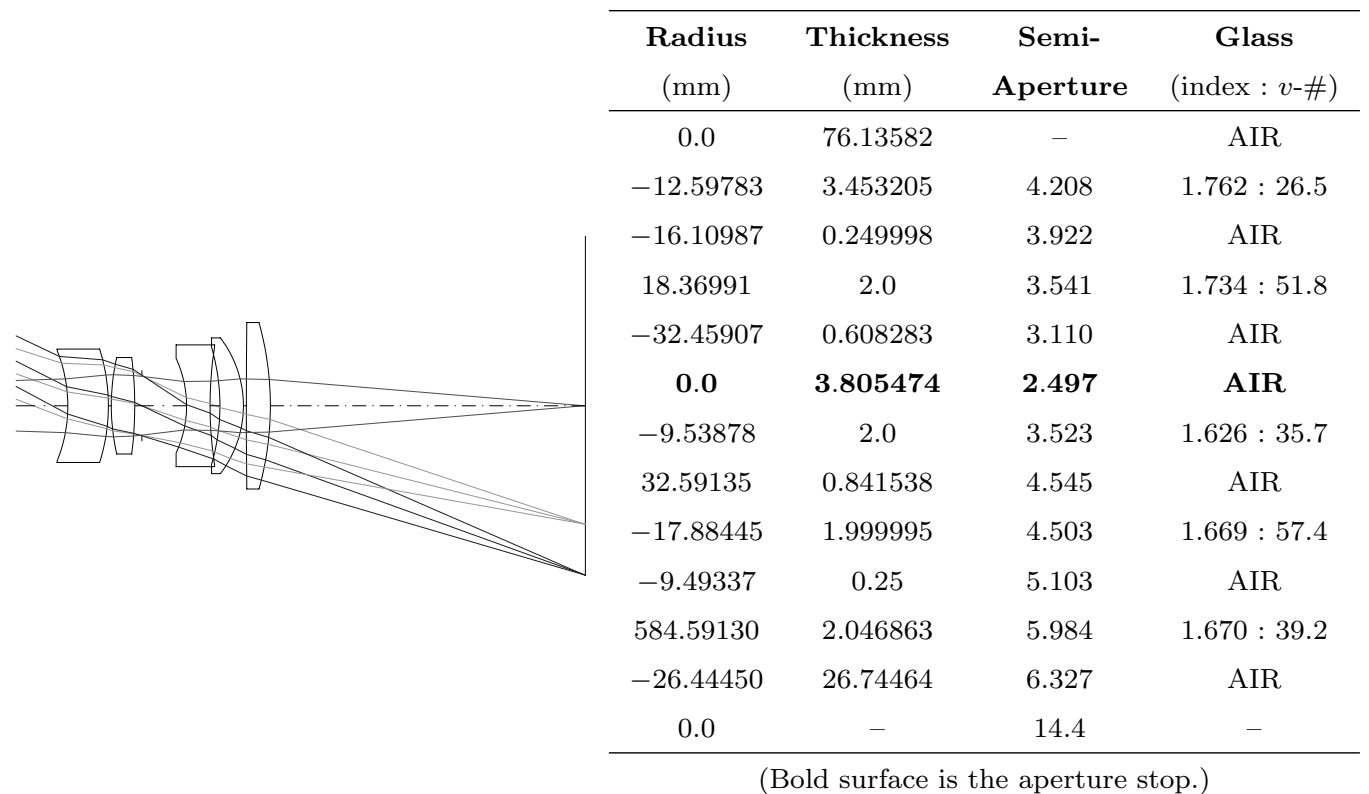

Fig. 8. Best system found by INO experts for the ILS problem.

within the $15 \mu \mathrm{m}$ prescribed for points at $0 \mathrm{~mm}$ on the object, the design can be considered to provide the desired image quality. But the energy diameters observed for points at $28 \mathrm{~mm}$ and $40 \mathrm{~mm}$ on the object indicate that the image is probably less well defined than expected near the borders.

For the EA experiments, the solutions are represented as vectors of 30 realvalued parameters. Table 4 summarizes these parameters along with the value transformations that are applied on the vector components of solutions. Note that this time, there is no specification on the glass indices and $v$-numbers. Original values for stop location $(\tilde{s})$, glass indices $\left(\tilde{n}_{j}\right)$, and glass $v$-numbers $\left(\tilde{v}_{j}\right)$ have been normalized using a triangular wave function in order to scale values in $[0,1]$. Over this normalization, glass indices and $v$-numbers have been transformed from a $\tilde{n}_{j} \in[0,1]$ and $\tilde{v}_{j} \in[0,1]$ square space into a quadrilateral space delimited by the $\left(\stackrel{\circ}{n}_{j}, \stackrel{\circ}{v}_{j}\right)$ corners at $(1.487,70.4),(1.620,60.3)$, $(1.744,44.7)$, and $(1.755,27.6)$. This domain includes indices and $v$-numbers of the most commonly used glasses in lens design. Contrary to the monochromatic quartet experiments, the EA is this time constrained to locate the stop on a lens surface, rather than let it float anywhere in the system. The stop location is the surface just at the left-hand side of the relative stop position given by parameter $s$. The distance between the last surface and the image plane is computed rather than evolved in order to get an in-focus paraxial image on the image plane. Finally, even if the specification on the $f$-number is rather lax, it was fixed to 4.0 for all of the EA runs.

The fitness measure used is similar to the one used for the monochromatic quartet experiments, with a single fitness real-value and a feasibility Boolean flag. If the original system is not feasible, the fitness value is computed as a 
Table 4

Parameters and value transformations for the ILS problem. Values marked by a tilde (i.e. $\tilde{x}$ ) are the original optimized parameter before the transformation, and values marked by a dot (i.e. $\stackrel{x}{)}$ ), as well as the stop location, are normalized within $[0,1]$ using a triangular wave function.

\begin{tabular}{|c|c|c|}
\hline Type & \# of Param. & Value Transformation \\
\hline Curvature & 10 & $c_{j}=0.025 \tilde{c}_{j}$ \\
\hline Thickness & 5 & $t_{j}=\left|20 \tilde{t}_{j}\right|+2$ \\
\hline Distance & 4 & $t_{j}=\left|20 \tilde{t}_{j}\right|$ \\
\hline Glass index & 5 & $\stackrel{\circ}{n}_{j}=\left\{\begin{array}{l}\left|\tilde{n}_{j}\right|-\left\lfloor\left|\tilde{n}_{j}\right|\right\rfloor \text { if }\left\lceil\left|\tilde{n}_{j}\right|\right\rceil \text { is odd } \\
\left\lceil\left|\tilde{n}_{j}\right|\right\rceil-\left|\tilde{n}_{j}\right| \text { otherwise }\end{array}\right.$ \\
\hline & & $n_{j}=0.135 \stackrel{\circ}{j}_{j}-0.133 \stackrel{\circ}{j}_{j}+0.122 \stackrel{\circ}{n}_{j} \stackrel{\circ}{v}_{j}+1.620$ \\
\hline$v$-number & 5 & $\stackrel{\circ}{v}_{j}=\left\{\begin{array}{l}\left|\tilde{v}_{j}\right|-\left\lfloor\left|\tilde{v}_{j}\right|\right\rfloor \text { if }\left\lceil\left|\tilde{v}_{j}\right|\right\rceil \text { is odd } \\
\left\lceil\left|\tilde{v}_{j}\right|\right\rceil-\left|\tilde{v}_{j}\right| \text { otherwise }\end{array}\right.$ \\
\hline & & $v_{j}=-32.7 \stackrel{\circ}{n}_{j}+10.1 \stackrel{\circ}{v}_{j}+7.0 \stackrel{\circ}{n}_{j} \stackrel{\circ}{j}_{j}+60.3$ \\
\hline Stop Location & 1 & $s=\left\{\begin{array}{l}|\tilde{s}|-\lfloor|\tilde{s}|\rfloor \text { if }\lceil|\tilde{s}|\rceil \text { is odd } \\
\lceil|\tilde{s}|\rceil-|\tilde{s}| \text { otherwise }\end{array}\right.$ \\
\hline
\end{tabular}

penalty sum:

$$
F_{1}=1000+\sum P_{a_{j}}+P_{y_{\mathrm{img}}}+P_{t t}+P_{\mathrm{vign}}+P_{t_{\mathrm{img}}}
$$

where the different $P_{x}$ are the penalties associated to the system constraints summarized in Table 5. Because these physical constraints are difficult to satisfy, some of them (i.e. semi-aperture, image size, and total track) have been relaxed in order to avoid tagging the lens system as not feasible when they are not satisfied. If the original system is feasible, the $75 \%$ encircled energy $E_{y_{\mathrm{obj}}}$ is computed from three points on the object plane $\left(y_{\mathrm{obj}}=0 \mathrm{~mm}\right.$, $y_{\mathrm{obj}}=28 \mathrm{~mm}$, and $y_{\mathrm{obj}}=40 \mathrm{~mm}$ ), taking the maximum value among the three diameters obtained. The $75 \%$ encircled energy is a measure to evaluate the quality of a lens system, different from the RMS blur spot used with the monochromatic quartet. The fitness value also includes the penalty value for the three relaxed constraints.

$$
F_{2}=\frac{\max _{j=\{0,28,40\}} E_{y_{\mathrm{obj}}=j}^{\mathrm{orig}}}{0.015}+\sum P_{a_{j}}+P_{y_{\mathrm{img}}}+P_{\mathrm{tt}}
$$

Then, the system is locally optimized for six seconds. If it is still feasible, the 
Table 5

Physical constraints of the ILS. Different $P_{x}$ penalties are set to zero when the associated constraints are satisfied. Constraints marked by a $\dagger$ do not affect feasibility of lens system when they are not satisfied. $a_{j}$ is the semi-aperture value of lens $j$. $l_{\text {vign }}$ is the number of surfaces where there is vignetting or that are not reached by ray tracing because of vignetting.

\begin{tabular}{|c|c|c|}
\hline Type & Constraint & Penalty \\
\hline Semi-aperture $^{\dagger}$ & $a_{j} \leq 15 \mathrm{~mm} \quad \forall j$ & $P_{a_{j}}=2 a_{j}-30$ \\
\hline Image size ${ }^{\dagger}$ & $\left|y_{\text {img }}\right| \in[12.4,16.4]$ & $P_{y_{\mathrm{img}}}=\left\{\begin{array}{c}\left(144 / y_{\mathrm{img}}\right)-10 \mathrm{~mm} \\
\text { if }\left|y_{\mathrm{img}}\right|<12.4 \\
0.694 y_{\mathrm{img}}-10 \mathrm{~mm} \\
\text { if }\left|y_{\mathrm{img}}\right|>16.4\end{array}\right.$ \\
\hline Total track ${ }^{\dagger}$ & $\sum t_{j} \leq 119 \mathrm{~mm}$ & $P_{t t}=\sum t_{j}-119$ \\
\hline Vignetting & $l_{\text {vign }}=0$ & $P_{\text {vign }}=1000 l_{\text {vign }}$ \\
\hline Image distance & $t_{\text {img }} \geq 0$ & $P_{t_{\mathrm{img}}}=-10 t_{\mathrm{img}}$ \\
\hline
\end{tabular}

fitness is recomputed as:

$$
F_{3}=\frac{\max _{j=\{0,28,40\}} E_{y_{\mathrm{obj}}=j}^{\mathrm{opt}}}{0.015}+\sum P_{a_{j}}+P_{y_{\mathrm{img}}}+P_{\mathrm{tt}}
$$

As with the monochromatic quartet, the $75 \%$ encircled energy diameter is a brittle quality criterion that is not computable for some unfeasible imaging lens systems. This again explain why distinct functions are used here as fitness measures for evaluating the quality of different systems.

The first experiments with the ILS problem are conducted using the $(\mu+$ $\lambda)$ non-isotropic SA-ES. Except for the initial mutation strategy parameter values that are set to $\sigma_{j}=1.0$, parameters are the same as those used for the monochromatic quartet: five populations of $\mu=50$ individuals, $\lambda=350$, two migrants, and minimum strategy parameter value of $\sigma_{j}=0.05$. Figure 9 presents the mutation and crossover algorithm used to generate new children for the actual experiments. This is different from the monochromatic quartet problem experiments, where only mutation was used. Figure 10 illustrates the best system generated over five $(\mu+\lambda)$ non-isotropic SA-ES evolutions of five $\mu=50$ demes. This system has a $75 \%$ encircled energy diameter of $11.68 \mu \mathrm{m}$. The averaged energy diameter obtained on the best systems found is 12.19 $\mu \mathrm{m}$ (standard deviation of $0.48 \mu \mathrm{m}$ ). Average computation time needed to run each evolution is a little less than 8 days (190 hours).

The image quality of the best imaging system found with $(\mu+\lambda)$ non-isotropic SA-ES is significantly better than the target value given in the specifications 
(1) Let $Y^{p 1}=\left(\left\{x_{1}^{p 1}, \sigma_{1}^{p 1}\right\},\left\{x_{2}^{p 1}, \sigma_{2}^{p 1}\right\}, \cdots,\left\{x_{n}^{p 1}, \sigma_{n}^{p 1}\right\}\right)$ and $Y^{p 2}=$ $\left(\left\{x_{1}^{p 2}, \sigma_{1}^{p 2}\right\},\left\{x_{2}^{p 2}, \sigma_{2}^{p 2}\right\}, \cdots,\left\{x_{n}^{p 2}, \sigma_{n}^{p 2}\right\}\right)$ be a copy of two randomly selected individuals taken from the parent population of size $\mu$, each individual being composed of $n$ pairs of $\{x, \sigma\}$ real values;

(2) Apply classical non-isotropic SA-ES mutation [4] to $Y^{p 1}$ and $Y^{p 2}$ individuals to generate the mutated $\bar{Y}^{p 1}$ and $\bar{Y}^{p 2}$ individuals;

(3) Create the individual $Y^{c}=\left(\left\{x_{1}^{c}, \sigma_{1}^{c}\right\},\left\{x_{2}^{c}, \sigma_{2}^{c}\right\}, \cdots,\left\{x_{n}^{c}, \sigma_{n}^{c}\right\}\right)$ with uniform crossover by setting each composing real value to $\left\{x_{j}^{c}, \sigma_{j}^{c}\right\}=$ $\left\{\bar{x}_{j}^{p 1}, \bar{\sigma}_{j}^{p 1}\right\}$, if $\mathcal{U}_{j}(0,1)<0.5$ or $\left\{x_{j}^{c}, \sigma_{j}^{c}\right\}=\left\{\bar{x}_{j}^{p 2}, \bar{\sigma}_{j}^{p 2}\right\}$, if $\mathcal{U}_{j}(0,1) \geq 0.5$ where $\left\{\bar{x}_{j}^{p 1}, \bar{\sigma}_{j}^{p 1}\right\}$ and $\left\{\bar{x}_{j}^{p 2}, \bar{\sigma}_{j}^{p 2}\right\}$ are composing values of the mutated $\bar{Y}^{p 1}$ and $\bar{Y}^{p 2}$ individuals, and $\mathcal{U}_{j}(0,1)$ are randomly generated numbers using an uniform distribution in $[0,1]$;

(4) Return $Y^{c}$ as the generated child.

Fig. 9. Mutation and crossover algorithm used to generate children in $(\mu+\lambda)$ non-isotropic SA-ES experiments for the ILS problem.

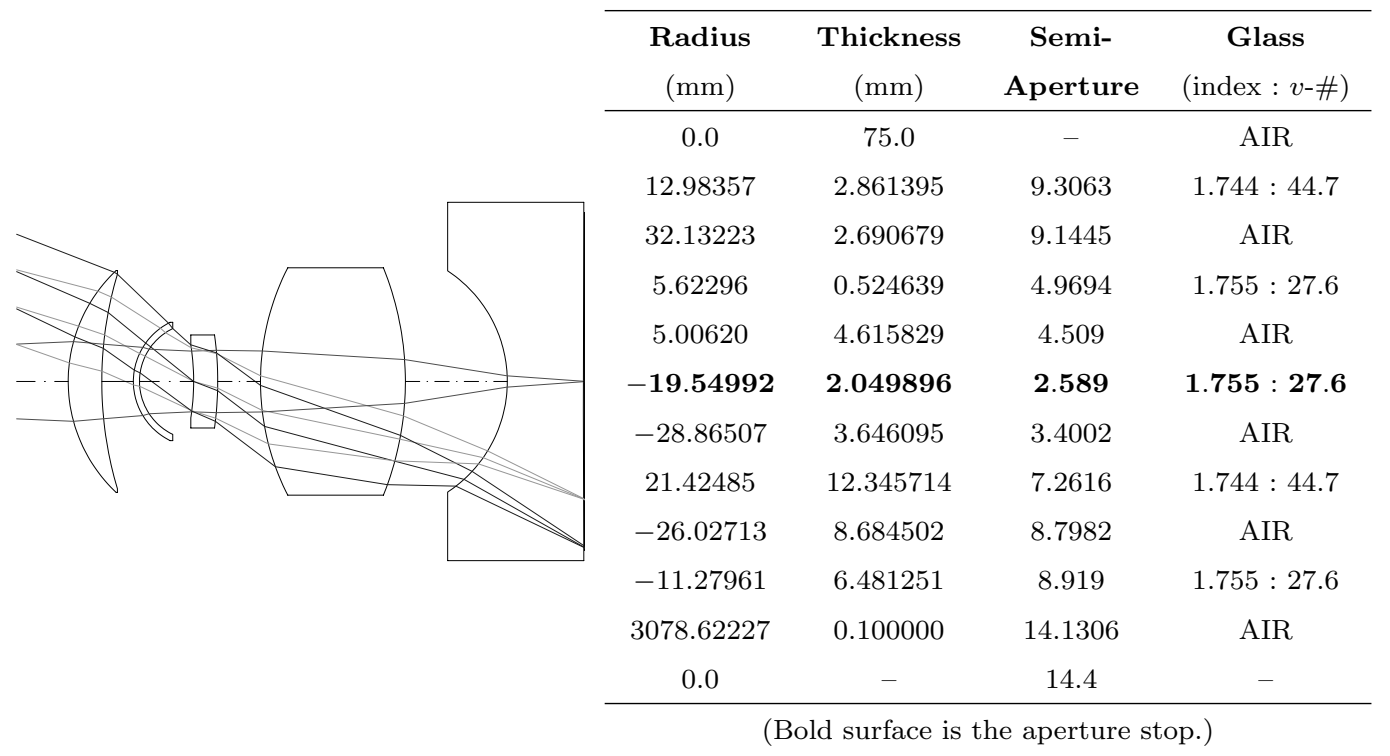

Fig. 10. Best lens system found with $(\mu+\lambda)$ non-isotropic SA-ES for the ILS problem (75\% encircled energy diameter of $11.68 \mu \mathrm{m})$.

(15 $\mu \mathrm{m})$. The imaging problem has strong physical constraints, and the optimization of the image quality is probably harder given these constraints. From a practical point of view, however, the second lens of the system is a fragile thin concentric meniscus that may introduce some assembly difficulties. Even though this type of consideration was not taken into account by the EA, additional constraints could be added to penalize such fragile designs.

An important point to note is that for all the five experiments with $(\mu+\lambda)$ non-isotropic SA-ES for the imaging problem, the best design belong to the same design class. This indicates a good repeatability capacity of $(\mu+\lambda)$ 


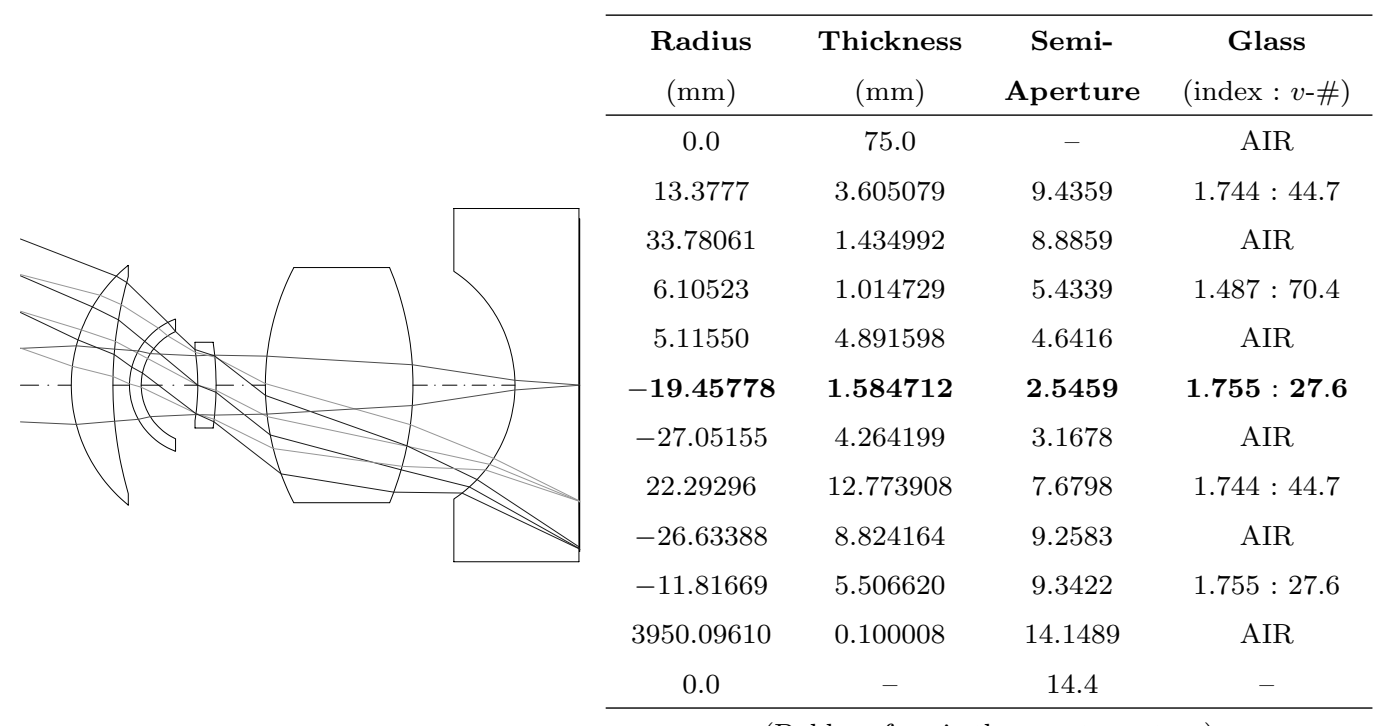

(Bold surface is the aperture stop.)

Fig. 11. Best lens system found with CMA-ES for the ILS problem (75\% encircled energy diameter of $12.05 \mu \mathrm{m})$.

non-isotropic SA-ES for the imaging problem.

For the second series of experiments, the CMA-ES algorithm was applied to the imaging problem. Parameters used are: a population of $\mu=10, \lambda=$ 50 children, runs of 500 generations, and individuals generated with random values uniformly distributed in $[-1,1]$. Figure 11 presents the best imaging system found with CMA-ES over five evolutions. The average of the $75 \%$ encircled energy diameter of the best system found for each evolution is 14.09 $\mu \mathrm{m}$ (standard deviation of $1.52 \mu \mathrm{m}$ ). Average computation time needed to run one CMA-ES evolution is 41 hours.

The best imaging system found with CMA-ES has a $75 \%$ encircled energy diameter of $12.05 \mu \mathrm{m}$, which is slightly more than the best design found with $(\mu+\lambda)$ non-isotropic SA-ES. Moreover, this design belongs to the same design class as the one obtained with $(\mu+\lambda)$ non-isotropic SA-ES.

\section{Multiobjective Optimization}

In the previous section, once the physical constraints of the system were satisfied, the lens system problem was formulated so that image quality could be optimized through the minimization of the $75 \%$ encircled energy diameter. But in real-life situations, the lens system designer may need to optimize other characteristics, such as system cost or assembly tolerance. In this section we investigate the use of multiobjective EC methods for simultaneous optimization of the image quality and relative cost in the context of the ILS 
problem.

The Pareto front for population-based optimization is the set of solutions that are not dominated by the other solutions of the population [25]. A solution is said to dominate another if all of its objective values are better or equal and if at least one objective value is better. The Pareto front of a population consists in the set of non-dominated solutions. Usual multiobjective EC methods use a selection operation that is based on the concept of Pareto dominance.

As a final series of experiments, two-objective EC optimizations are conducted for the ILS problem. The first objective is to minimize the fitness measure presented in Equations 7, 8, and 9. The second objective is to minimize the total cost of the lenses. Regarding the multiobjective fitness, it should be noted that a feasible solution is always considered as dominating a non-feasible one, notwithstanding the respective objective values. The cost is evaluated using the list of relative prices per gram for different types of glass (see Table 6; this list is a subset of the SCHOTT optical glass catalog [38]; SCHOTT glasses are widely used in the industry). The relative cost per gram for an arbitrary glass is taken from the nearest glass of Table 6 using the following distance measure:

$$
d=\sqrt{\left(n_{a}-n_{l}\right)^{2}+\frac{\left(v_{a}-v_{l}\right)^{2}}{10000}}
$$

where $n_{a}$ and $v_{a}$ are the refractive index and $v$-number of the arbitrary glass, and $n_{l}$ and $v_{l}$ those of a glass in the list of Table 6 .

Experiments have been conducted using a multiobjective variant of non-isotropic SA-ES. The $(\mu+\lambda)$ replacement strategy is changed for the NSGA-II (Non-Dominated Sort Genetic Algorithm 2) [39], more commonly used within the GA paradigm. It is in some way similar to the $(\mu+\lambda)$ approach, but with $\mu=\lambda$ and the particularity that individuals are selected for a new generation using a sophisticated multiobjective sort of the parents and children population based on dominance and niching. Population size was set to 500 individuals, runs were conducted for 500 generations, and children were generated using the same crossover and mutation operators as previously (see Fig. 9). Five different evolution runs were conducted using these parameters for multiobjective optimization of the ILS problem.

The selection of the best-of-run individuals for multiobjective optimization is not as straightforward as for single objective optimization, where we simply selected the individual with the best fitness value. In the present case, the best-of-run individual is selected as the individual on the Pareto front with the smallest relative price that has a $75 \%$ encircled energy diameter under $15 \mu \mathrm{m}$. The overall best individual over the five evolution runs is selected 
Table 6

List of relative cost per gram for common glasses typically used in lens system design (subset of SCHOTT optical glass catalog [38]). NBK7 glass is used as reference with a relative cost of 1.0 .

\begin{tabular}{|c|c|c|c|c|c|c|c|}
\hline Glass & Index & $v$-number & Price/g & Glass & Index & $v$-number & Price/g \\
\hline F2 & 1.62004 & 36.37 & 1.6 & NLAK10 & 1.72003 & 50.62 & 4.5 \\
\hline $\mathrm{F} 4$ & 1.61659 & 36.63 & 2.5 & NLAK12 & 1.6779 & 55.2 & 3 \\
\hline F5 & 1.60342 & 38.03 & 2 & NLAK14 & 1.6968 & 55.41 & 3 \\
\hline K10 & 1.50137 & 56.41 & 2.5 & NLAK21 & 1.64049 & 60.1 & 3.5 \\
\hline $\mathrm{K} 7$ & 1.51112 & 60.41 & 2 & NLAK22 & 1.65113 & 55.89 & 3.5 \\
\hline KZFSN4 & 1.6134 & 44.29 & 3 & NLAK33 & 1.75398 & 52.43 & 11.5 \\
\hline KZFSN5 & 1.65412 & 39.63 & 3 & NLAK7 & 1.6516 & 58.52 & 3.5 \\
\hline LAFN7 & 1.7495 & 34.95 & 5 & NLAK8 & 1.713 & 53.83 & 3 \\
\hline LF5 & 1.58144 & 40.85 & 2 & NLAK9 & 1.691 & 54.71 & 5 \\
\hline LLF1 & 1.54814 & 45.75 & 2.5 & NSF1 & 1.71736 & 29.62 & 4 \\
\hline NBAF10 & 1.67003 & 47.11 & 3 & NSF10 & 1.72828 & 28.53 & 4 \\
\hline NBAF3 & 1.58272 & 46.64 & 3.5 & NSF15 & 1.69892 & 30.2 & 3.5 \\
\hline NBAF4 & 1.60568 & 43.72 & 4 & NSF5 & 1.67271 & 32.25 & 3.5 \\
\hline NBAF51 & 1.65224 & 44.96 & 3 & NSF64 & 1.70591 & 30.23 & 3.5 \\
\hline NBAF52 & 1.60863 & 46.6 & 3 & NSF8 & 1.68894 & 31.31 & 3.5 \\
\hline NBAK1 & 1.5725 & 57.55 & 2 & NSK10 & 1.62278 & 56.98 & 4 \\
\hline NBAK2 & 1.53996 & 59.71 & 2 & NSK11 & 1.56384 & 60.8 & 3 \\
\hline NBAK4 & 1.56883 & 55.98 & 1.5 & NSK14 & 1.60311 & 60.6 & 2.5 \\
\hline NBALF4 & 1.57956 & 53.87 & 3 & NSK15 & 1.62296 & 58.02 & 2.5 \\
\hline NBALF5 & 1.54739 & 53.63 & 2.5 & NSK16 & 1.62041 & 60.32 & 3 \\
\hline NBASF2 & 1.66446 & 36 & 3 & NSK2 & 1.60738 & 56.65 & 2 \\
\hline NBASF64 & 1.704 & 39.38 & 3 & NSK4 & 1.61272 & 58.63 & 6 \\
\hline NBK10 & 1.49782 & 66.95 & 2 & NSK5 & 1.58913 & 61.27 & 1.5 \\
\hline NBK7 & 1.5168 & 64.17 & 1 & NSSK2 & 1.62229 & 53.27 & 5 \\
\hline NF2 & 1.62005 & 36.43 & 2 & NSSK5 & 1.65844 & 50.88 & 3 \\
\hline NK5 & 1.52249 & 59.48 & 2 & NSSK8 & 1.61773 & 49.83 & 3.5 \\
\hline NKF9 & 1.52346 & 51.54 & 2 & NZK7 & 1.50847 & 61.19 & 3 \\
\hline NKZFS4 & 1.61336 & 44.49 & 11 & SF1 & 1.71736 & 29.51 & 2.5 \\
\hline NLAF2 & 1.74397 & 44.85 & 3.5 & SF10 & 1.72825 & 28.41 & 2 \\
\hline NLAF3 & 1.717 & 47.96 & 6 & SF15 & 1.69895 & 30.07 & 2.5 \\
\hline NLAF7 & 1.7495 & 34.82 & 5.5 & SF5 & 1.6727 & 32.21 & 5 \\
\hline
\end{tabular}

as the best-of-run individual with the smallest relative price. Figure 12 gives the Pareto front of the last generation for the overall best individual which is summarized in Fig. 13. It has a $75 \%$ encircled energy diameter of $15.0 \mu \mathrm{m}$, and a relative price of 50.96 . The average $75 \%$ encircled energy diameter of the best-of-run individual for the five independent runs is $14.9 \mu \mathrm{m}$ (standard deviation of $0.14 \mu \mathrm{m}$ ), and the average relative cost is 95.9 (standard deviation of 29.7). The evolutions where conducted on a different processor than for the previous experiments (Pentium M $1.9 \mathrm{GHz}$ laptop PC) and the average CPU time needed on this computer was a little less than 25 hours per evolution. 


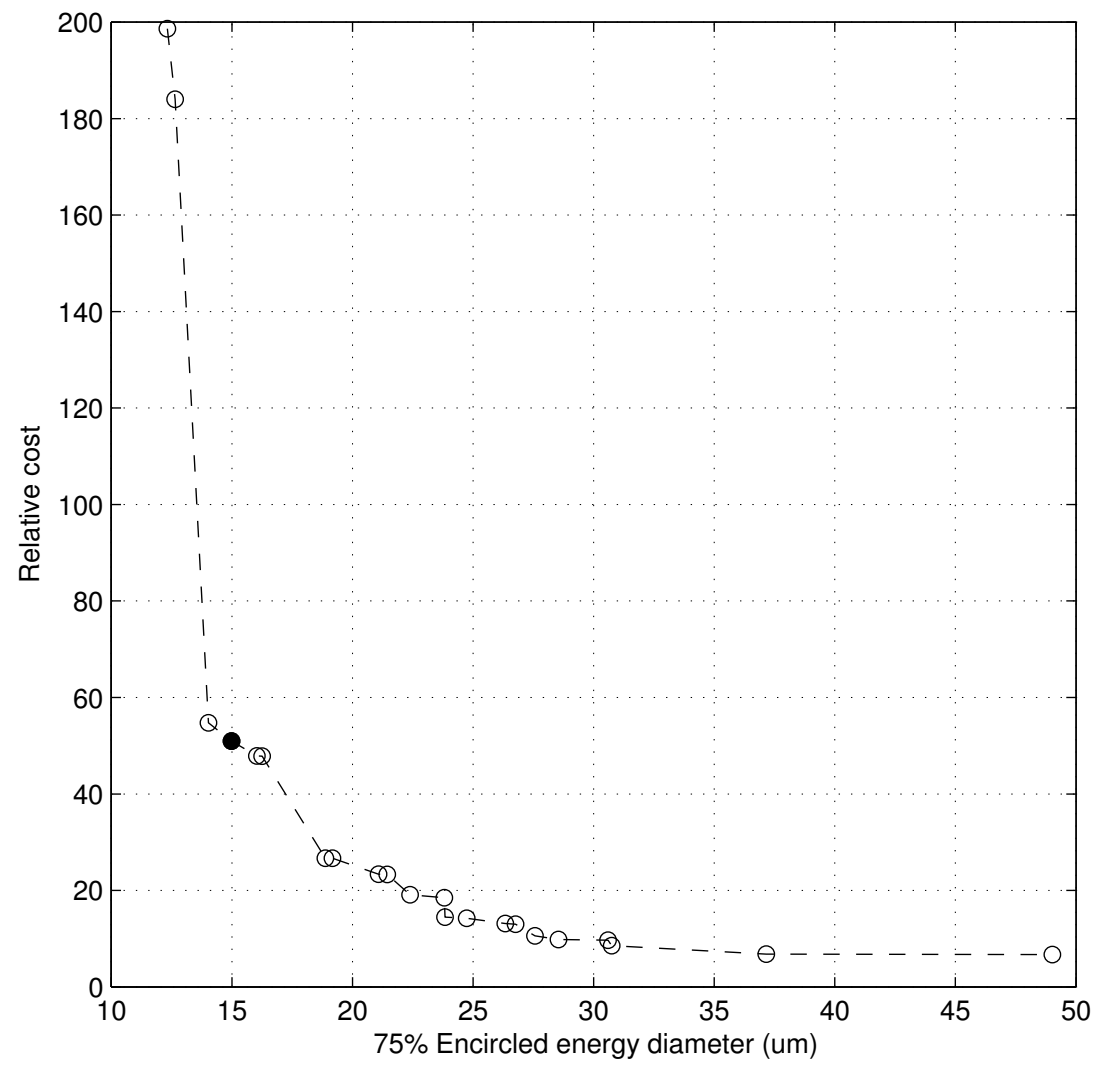

Fig. 12. Pareto front of the experiments with the overall best individual. Individuals with a first objective value that corresponds to a $75 \%$ encircled energy diameter higher than $50 \mu \mathrm{m}$ (when not penalized) have been omitted. The selected best-of-run solution is designated by the black dot.

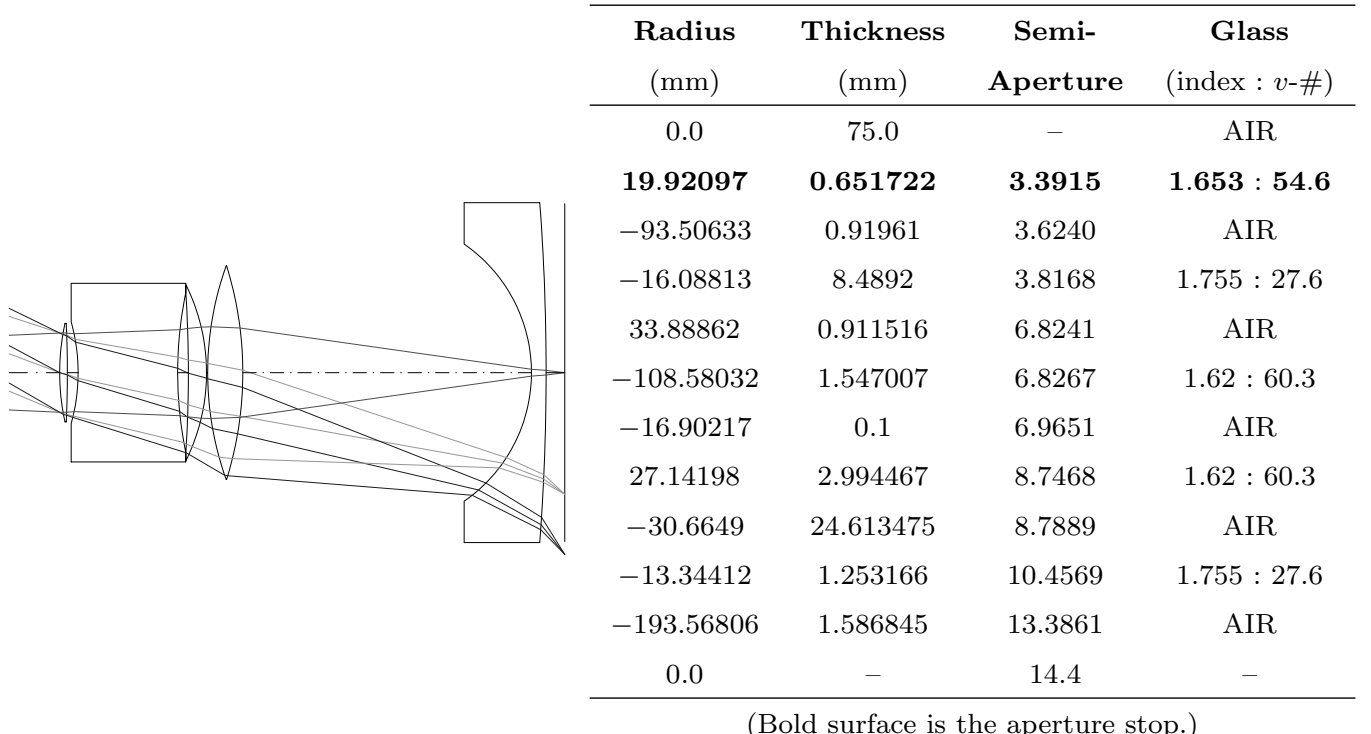

Fig. 13. Best lens system found with multiobjective NSGA-II non-isotropic SA-ES for the ILS problem ( $75 \%$ encircled energy diameter of $15.0 \mu \mathrm{m}$ and relative cost of $50.96)$. 
In comparison, the INO imaging system has a relative cost of 10.4, which is almost five times smaller than the price of the best lens system found with the NSGA-II algorithm. But, it should be noticed that the maximum $75 \%$ encircled energy diameter for the INO system is in fact $33.3 \mu \mathrm{m}$, which is more than twice the image quality of the best evolved lens system. Taking a closer look at the Pareto front, we can see that the solution of comparable image quality has a maximum $75 \%$ encircled energy diameter of $30.7 \mu \mathrm{m}$, with a relative cost of only 8.53 . This is $18 \%$ less than the INO system's relative cost. On the other hand, the system with the nearest relative cost on the same Pareto front (relative cost of 9.80) has a maximum $75 \%$ encircled energy diameter of $28.5 \mu \mathrm{m}$, which is significantly better than for the INO system. This illustrates the advantages of using an evolutionary multiobjective optimization based on Pareto optimality. It allows the selection of the most interesting solution for a given problem in the knowledge of the different possible trade-offs.

In a practical context, the method used to select the best-of-run individuals for all of the multiobjective experiments makes sense. For the imaging problem, once the $75 \%$ encircled energy diameter is less than $15 \mu \mathrm{m}$, other considerations can be taken into account during the design process. In the present case, the total relative cost is used as a second objective. Taking a look at the best lens system found, it can be seen that it is in a class of design close to those previously presented, while being made of inexpensive glasses. Moreover, contrary to the two previous EA-found systems, the best multiobjective design does not have a fragile thin meniscus as second lens (starting from the left).

\section{Conclusion}

Because of its very complex nature, where intricate physical constraints must be dealt with, it would be surprising that human expertise could be completely removed from lens system design any time soon. However, we have shown in this paper that evolutionary computations can be human competitive [40] for real-world lens design, and thus advocate that it should be part of every lens designer's toolbox. Current specialized CAD tools offer mostly local optimization, but only after a human has produced a draft design. By combining an evolutionary algorithm with a local search algorithm, and through the use of multiobjective optimization, we were able to find solutions that sometimes outperform those produced by human experts.

More specifically two evolution strategies were studied, namely $(\mu+\lambda)$ nonisotropic SA-ES and CMA-ES, each hybridized with a damped least square algorithm. The goal of this hybrid approach is to take advantage of both the exploratory nature of EC and the exploitation capabilities of the finely tuned local search algorithm. To the best of our knowledge, a similar approach 
has only been explored in [16], but with genetic algorithm evolved solutions representing zero-power transformations of an initially simple lens system, not by working directly on the system parameters.

Results obtained in this work are for a benchmark problem, called the monochromatic quartet, that was initially introduced as a friendly competition between human experts, and a real imaging problem that was presented to human experts of the INO research center. For both problems, two series of experiments were conducted using respectively the $(\mu+\lambda)$ non-isotropic SA-ES and the CMA-ES algorithms. For the first problem, the best evolved solution using SA-ES was found to outperform the best human solution by over $23 \%$. This evolved design satisfies all of the problem specifications, but the solution itself is not practical because of its extreme dimensions. Nevertheless, it invalidates the common belief that the two categories of designs found by human experts are global optimums. Using the CMA-ES algorithm, one of these two design classes was re-discovered, with a level of performance in between the worst and best human expert.

For the second problem, a real-world application was tackled with severe physical constraints. With a best effort of five man-days of work, the INO experts designed a system that was surpassed by both the SA-ES and CMA-ES algorithms. The only weak part of these evolved designs, in both cases, is that one of the lenses is very thin, in practice probably too thin to be fabricated and assembled at reasonable cost. But since no specification was given against such a solution, the EA approach cannot be discredited for converging towards such a lens. In order to evolve lens systems that satisfy practical considerations, the common sense of optical design must be implemented into a set of general constraints.

A second objective was then added in the evolution process: the minimization of the cost of raw lens material. Using NSGA-II for multiobjective optimization, solutions were found that both outperformed the INO design on image quality at equivalent cost, and cost less at equivalent image quality.

In general, the non-isotropic SA-ES algorithm was found to explore more thoroughly the solution space, and thus to discover better solutions, but the CMA-ES algorithm tends to converge much more rapidly to solutions that are almost as good as those of SA-ES. This results mostly from the ability of CMA-ES to work with much smaller populations, whereas non-isotropic SA-ES requires larger populations to adequately re-estimate its mutation parameters. 


\section{Acknowledgements}

This work was supported by NSERC-Canada and FQRNT-Québec.

\section{References}

[1] T. Bäck, D. B. Fogel, Z. Michalewicz (Eds.), Evolutionary Computation 1: Basic Algorithms and Operators, Institute of Physics Publishing, Bristol, UK, 2000.

[2] T. Bäck, U. Hammel, H.-P. Schwefel, Evolutionary computation: comments on the history and current state, IEEE Transactions on Evolutionary Computation 1 (1) (1997) 3-17.

[3] J. R. Koza, M. A. Keane, J. Yu, F. H. Bennett III, W. Mydlowec, Automatic creation of human-competitive programs and controllers by means of genetic programming, Genetic Programming and Evolvable Machines 1 (1/2) (2000) $121-164$.

[4] H.-G. Beyer, H.-P. Schwefel, Evolution strategies: a comprehensive introduction, Natural Computing 1 (1) (2002) 3-52.

[5] A. Auger, Contributions théoriques et numériques à l'optimisation continue par algorithmes évolutionnaires, Ph.D. thesis, Université Paris 6 (2004).

[6] I. Rechenberg, Evolutionsstrategie, Friedrich Frommann Verlag (Günther Holzboog KG), Stuttgart, 1973.

[7] N. Hansen, S. D. Mueller, P. Koumoutsakos, Reducing the time complexity of the derandomized evolution strategy with covariance matrix adaptation (CMAES), Evolutionary Computation 11 (1) (2003) 1-18.

[8] N. Hansen, A. Ostermeier, Completely derandomized self-adaptation in evolution strategies, Evolutionary Computation 9 (2) (2001) 159-195.

[9] N. Hansen, The CMA evolution strategy: A tutorial, http://www.bionik. tu-berlin.de/user/niko/cmatutorial.pdf (2005).

[10] D. C. O'Shea, Elements of Modern Optical Design, John Wiley and Sons, 1985.

[11] D. Sturlesi, D. C. O'Shea, Future of global optimization in optical design, in: Proc. of the Intl. Lens Design Conference (ILDC 1990), Vol. 1354 of Proc. of SPIE, 1990, pp. 54-68.

[12] G. W. Forbes, A. E. W. Jones, Towards global optimization with adaptive simulated annealing, in: Proc. of the Intl. Lens Design Conference (ILDC 1990), Vol. 1354 of Proc. of SPIE, 1990, pp. 144-153.

[13] G. K. Hearn, Pratical use of generalized simulated annealing optimization on microcomputers, in: Proc. of the Intl. Lens Design Conference (ILDC 1990), Vol. 1354 of Proc. of SPIE, 1990, pp. 186-193. 
[14] M. Isshiki, Global optimization with escape function, in: Proc. of the Intl. Optical Design Conference (IODC 1998), Vol. 3482 of Proc. of SPIE, 1998, pp. 104-109.

[15] M. Walk, J. Niklaus, Some remarks on computer-aided design of optical lens systems, Journal of Optimization Theory and Applications 59 (2) (1988) 173181.

[16] E. Betensky, Postmodern lens design, Optical Engineering 32 (8) (1993) 17501756.

[17] J. M. Holland, Adaptation in Natural and Artificial Systems, University of Michigan Press, Ann Arbor, MI, 1975.

[18] M. Mitchell, An Introduction to Genetic Algorithms, Complex Adaptive Systems, MIT-Press, Cambridge, 1996.

[19] S. Banerjee, L. Hazra, Experiments with a genetic algorithm for structural design of cemented doublets with prespecified aberration targets, Applied Optics 40 (34) (2001) 6265-6273.

[20] S. Chatterjee, L. Hazra, Structural design of cemented triplets by genetic algorithm, Optical Engineering 43 (2) (2004) 432-440.

[21] I. Ono, S. Kobayashi, K. Yoshida, Global and multi-objective optimization for lens design by real-coded genetic algorithms, in: Proc. of Intl. Optical Design Conference (IODC 1998), Vol. 3482 of Proc. of SPIE, 1998, pp. 110-121.

[22] D. Vasiljević, Classical and Evolutionary Algorithms in the Optimization of Optical Systems, Kluwer Academic Publishers, 2002.

[23] D. Vasiljević, J. Golobić, Comparison of the classical dumped least squares and genetic algorithm in the optimization of the doublet, in: First Online Workshop on Soft Computing, 1996, pp. 200-204.

[24] D. Vasiljević, Optimization of the Cooke triplet with various evolution strategies and damped least squares, in: Proc. of the SPIE Conf. on Optical Design and Analysis Software, Vol. 3780 of Proc. of SPIE, 1999, pp. 207-215.

[25] C. A. C. Coello, D. A. V. Veldhuizen, G. B. Lamont, Evolutionary Algorithms for Solving Multi-Objective Problems, Kluwer Academic Publishers, 2002.

[26] X. Chen, K. Yamamoto, An experiment in genetic optimization in lens design, Journal of Modern Optics 44 (9) (1997) 1693-1702.

[27] K. E. Moore, Algorithm for global optimization of optical systems based upon genetic competition, in: Proc. of the SPIE Conf. on Optical Design and Analysis Software, Vol. 3780 of Proc. of SPIE, 1999, pp. 104-109.

[28] J. Beaulieu, C. Gagné, M. Parizeau, Lens system design and re-engineering with evolutionary algorithms, in: Proc. of the Genetic and Evolutionary Computations COnference (GECCO 2002), New York, NY, USA, 2002, pp. $155-162$. 
[29] D. C. O'Shea, The monochromatic quartet: A search for the global optimum, in: Proc. of the Intl. Lens Design Conference (ILDC 1990), Vol. 1354 of Proc. of SPIE, 1990, pp. 548-554.

[30] Y. Nagata, The lens design using the CMA-ES algorithm, in: Proc. of the Genetic and Evolutionary Computations COnference (GECCO 2004), Vol. 3103 of LNCS, 2004, pp. 1189-1200.

[31] S. H. Al-Sakran, J. R. Koza, L. W. Jones, Automated re-invention of a previously patented optical lens system using genetic programming, in: Proc. of the European Conference on Genetic Programming (EuroGP 2005), Vol. 3447 of LNCS, 2005, pp. 25-37.

[32] J. R. Koza, S. H. Al-Sakran, L. W. Jones, Automated re-invention of six patented optical lens system using genetic programming, in: Proc. of the Genetic and Evolutionary Computations COnference (GECCO 2005), Washington, DC, USA, 2005, pp. 1953-1960.

[33] J. R. Koza, David Andre, F. H. Bennett III, M. Keane, Genetic Programming 3: Darwinian Invention and Problem Solving, Morgan Kaufman, 1999.

[34] Optical Research Associates, CODE V: Optical design, fabrication, and analysis software, http://www.opticalres.com/cv/cvprodds_f.html (2006).

[35] P. Moscato, On Evolution, Search, Optimization, Genetic Algorithms and Martial Arts: Towards Memetic Algorithms, Tech. Rep. Caltech Concurrent Computation Program, Report. 826, California Institute of Technology, Pasadena, California, USA (1989).

[36] Y. S. Ong, M. H. Lim, N. Zhu, K. W. Wong, Classification of adaptive memetic algorithms: A comparative study, IEEE Transactions On Systems, Man and Cybernetics, Part B 36 (1) (2006) 141-152.

[37] C. Gagné, M. Parizeau, Genericity in evolutionary computation software tools: Principles and case-study, International Journal on Artificial Intelligence Tools 15 (2) (2006) 173-194.

[38] SCHOTT AG, Optical glass catalog, http://www.schott.com/optics_ devices/english/products/flash/abbediagramm_flash.html (2006).

[39] K. Deb, A. Pratap, S. Agarwal, T. Meyarivan, A Fast and Elitist Multiobjective Genetic Algorithm: NSGA-II, IEEE Transactions on Evolutionary Computation 6 (2) (2002) 182-197.

[40] J. R. Koza, M. A. Keane, M. J. Streeter, W. Mydlowec, J. Yu, G. Lanza, Genetic Programming IV: Routine Human-Competitive Machine Intelligence, Kluwer Academic Publishers, 2003. 